\title{
Ein Gerät zur Entnahme ungestörter Sediment-Grossproben in Seen
}

Von Heinz AmbüHL

Eidgenössische Anstalt für Wasserversorgung, Abwasserreinigung und Gewässerschutz an der ETH Zürich, Direktion: Prof. Dr. O. JAAG. 


\section{Allgemeines}

Wohl die meisten Schlammlote, welche heute zur Erforschung der Sedimente der Binnenseen verwendet werden, liefern zwar Bohrkerne von mitunter beachtlicher Länge, aber nur mit einem verhältnismässig bescheidenen Durchmesser. Dieser genügt für sedimentstratigraphische Arbeiten, für mikrobiologische Untersuchungen oder auch für Pollenanalysen sehr wohl. Zur Prüfung chemischer Gleichgewichtsreaktionen, z.B. im Zusammenhang mit der Speicherung bzw. Freisetzung von Nährstoffen, reichen die gebräuchlichen Durchmesser (18 bis $34 \mathrm{~mm}$ ) indessen nicht aus, da sich zwischen der Sedimentfläche und der überstehenden Wassersäule ein allzu ungünstiges Verhältnis ergibt, welches den Aussagewert dieses Modells herabsetzt. Um solche Nachteile zu verringern, haben wir ein Gerät konstruiert, das erlaubt, ungestörte Proben mit einem Durchmesser von $112 \mathrm{~mm}$ zu entnehmen (Aussendurchmesser der Probenröhre $120 \mathrm{~mm}$ ).

Es handelt sich um eine Kombination von Schlammlot und Schlammgreifer. Das Tiefensediment unserer Binnenseen ist in sozusagen allen Fällen von zähflüssiger bis teigartiger Beschaffenheit; eine Probe, welche am oberen Ende des Lotrohres durch ein Vakuumventil gehalten wird [1,2], müsste bei grösseren Rohrdurchmessern nach unseren Erfahrungen beim Hieven als kompakter Schlammzylinder aus dem (unten offenen) Rohr hinausrutschen oder ausfliessen. Diese, Gefahr ist bei engen Rohren infolge der spezifisch grösseren Mantelreibung wesentlich kleiner. Es musste deshalb ein System gewählt werden, das einen sicheren Verschluss des unteren Rohrendes gewährleistet. Damit lassen sich auch die bekannten Schwierigkeiten umgehen, welche sich sonst beim Bergen des gefüllten Lotes häufig ergeben: Bevor das Rohr über die Wasseroberfläche gehoben wird, muss es, falls der Bohrkern nicht aus sehr kompaktem und deshalb selber als Dichtung wirkenden Material besteht, unten rasch mit einem Stopfen verschlossen werden. Dabei wird aber der Bohrkern im Rohr sehr leicht verschoben, was eine Verschleppung oder schiefe Verlagerung der Schichten und vor allem Schäden an der Schlammoberfläche zur Folge hat. Dies kann durch einen selbst- 


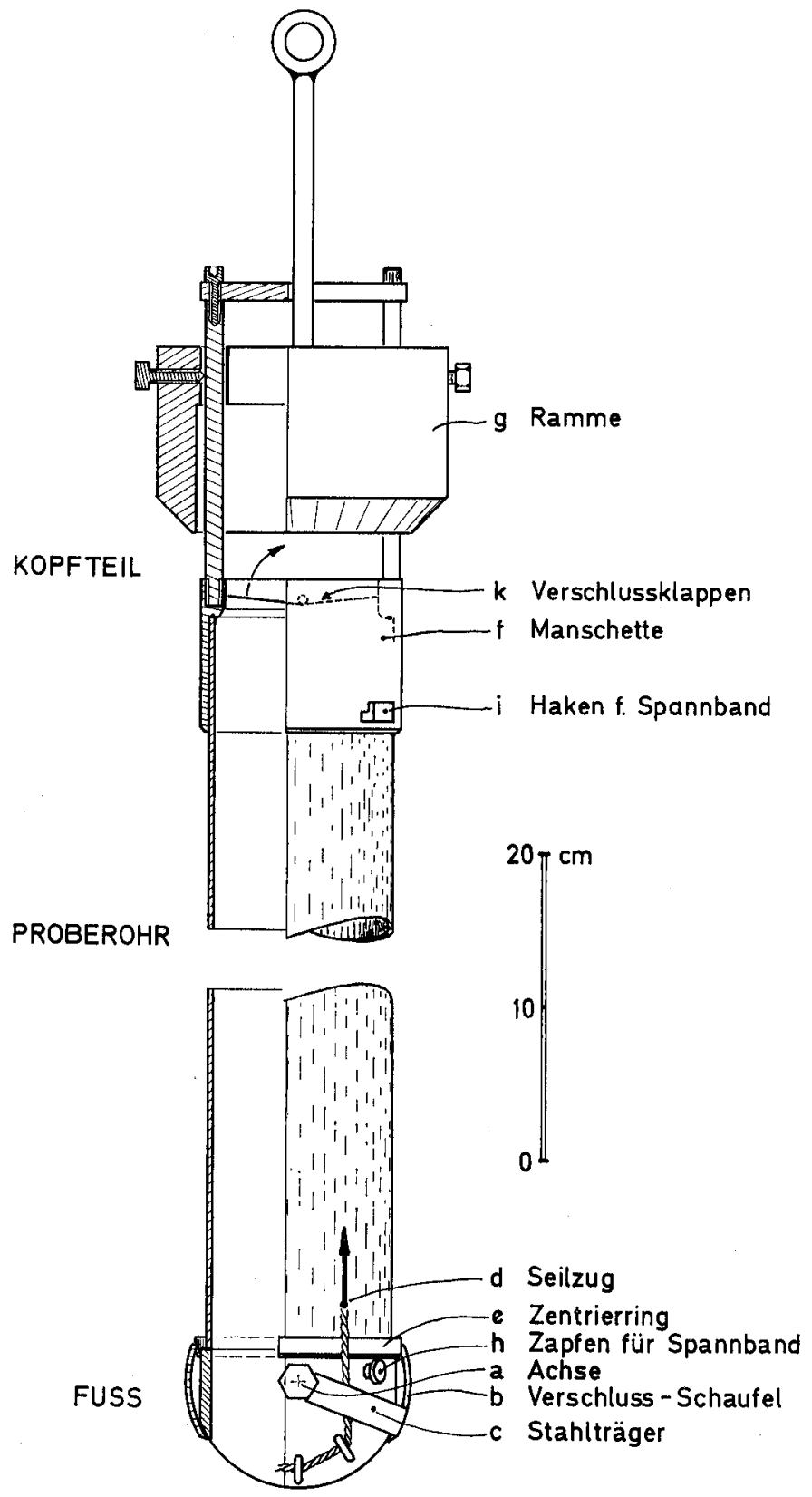

Abb. 1. Schema des Gerätes. Linke Hälfte als Längsschnitt, rechte Hälfte als Ansicht.

tätigen, noch vor dem Hieven des Gerätes in Funktion tretenden Verschluss vermieden werden. Freilich ergeben sich daraus andere Erschwernisse. So kann das gefüllte Proberohr nicht, wie bei den obenerwähnten Geräten, einfach an seinem oberen Ende 
aus seiner Halterung gelöst werden, um sofort zur Verfügung zu stehen, sondern der Schliessmechanismus muss durch einen anderen Verschluss ersetzt werden, um das Gerät für weitere Verwendung frei zu bekommen. Dies erfordert eine zusätzliche Einrichtung, welche gestattet, den Schneid- und Verschlussfuss vom Proberohr zu trennen, ohne den Bohrkern, der an der Wasser-Schlamm-Grenze besonders empfindlich ist, $\mathrm{zu}$ verletzen.

\section{Beschreibung des Gerätes}

Das Bohrgerät besteht, wie aus den Abbildungen 1 und 2 ersichtlich ist, im wesentlichen aus 4 Teilen, nämlich (von unten nach oben) aus

- dem Schneidfuss mit Verschlußschaufeln,

- dem Proberohr,

- dem Kopfteil mit Rammgewicht und

- dem Auslöser.

Der Fuss hat, wie das darüberstehende Rohr aus Plexiglas, einen inneren Durchmesser von $112 \mathrm{~mm}$. Er ist aus $130 \mathrm{~mm}$ starkem Stahlrohr gebaut, auf der oberen Seite plan abgedreht und auf der unteren in Form eines liegenden Kreiszylinders mit einem Radius $\mathrm{R}=2 D / \sqrt{3}(\mathrm{D}=$ Durchmesser des Fusses bzw. Höhe eines gleichseitigen Dreiecks mit der Seite $R$ ) abgeschnitten. Die Axe dieses Zylinders (Abb. 1, a) bildet gleichzeitig die Drehachse der beiden Verschlußschaufeln $(b)$ (ähnlich den Bodengreifern von EKMAN [3] oder von AUERBACH [4]), welche aus Stahlrohrstücken entsprechender Krümmung ausgeschnitten sind. Diese Schaufeln sind mit starken, festgeschweissten Stahlbändern am Fuss drehbar gelagert (c). Damit sie nicht von selbst zufallen, sondern namentlich während des Absenkens zum Seegrund offenstehen, sind in den Lagern $(a)$ Federscheiben eingefügt, deren Reibung für die Fixierung in offener Position ausreicht. Geschlossen werden die Schaufeln durch Seilzüge $(d)$. In das obere, plane Ende des Fusses ist eine Nut eingedreht, in die ein Dichtungsring (O-Ring) aus 2,5 mm starker Gummisaite eingelegt ist.

Das kreisrunde Proberohr besteht aus Acrylglas ("Plexiglas», "Perspex») in der handelsüblichen Abmessung von $120 \mathrm{~mm}$ Aussen- bzw. $112 \mathrm{~mm}$ Innendurchmesser. Die Länge kann beliebig gewählt werden; in unserem Prototyp wurde eine Länge von $950 \mathrm{~mm}$ verwendet. Solch weite Rohre sind ziemlich heikel zu bearbeiten. Angesichts der Bruchgefahr, welche bei unzweckmässiger Behandlung trotz den sonst hervorragenden Materialeigenschaften doch immer besteht, und angesichts des recht hohen Preises lassen wir die Rohre direkt vom Lieferanten auf ihre Gebrauchslänge zuschneiden und verzichten auf jede weitere Bearbeitung. Das Proberohr ist somit lediglich an beiden Enden plan abgeschnitten; die Dichtungen befinden sich an den Metallteilen. Um das Gerät für weitern Gebrauch wieder freizukriegen, muss die Probe vom Fussteil abgetrennt werden. Dies ist ohne Verlust oder Nachrutschung durch Schneiden mit einem dünnen Blech, zwischen Fussteil und Plexiglas eingeschoben, möglich, setzt aber voraus, dass beide Teile stumpf aufeinanderstehen, ohne eingedrehte Führungsnuten oder dergleichen. Allerdings wird das Rohr, um ihm doch einen sicheren Halt zu geben, mit Hilfe eines beweglichen Führungsringes (e), der gleichzeitig als oberer Anschlag für die Verschlußschaufeln wirkt, auf dem Fussteil bzw. auf der O-Ring-Dichtung zentriert. 


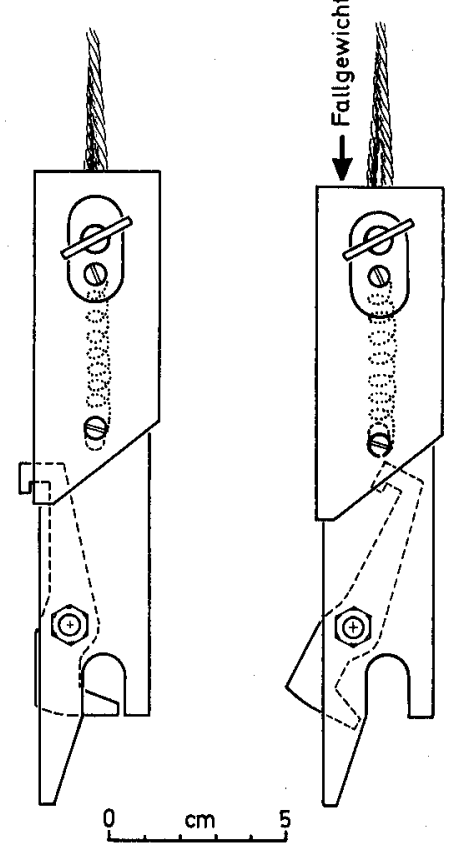

Abb. 2. Auslösemechanismus, links gespannt, rechts geöffnet. Material: Rechteckiges Stahlrohr $35 \times 25 \times 2$ und $30 \times 20 \times 2 \mathrm{~mm}$.

Der Kopfteil besteht aus einer $10 \mathrm{~cm}$ langen Manschette, welche auf das Proberohr aufgesetzt wird und an ihrem inneren Ende in einer eingedrehten Nut einen O-Ring trägt $(f)$. Dieser dient hier nicht als Dichtung, sondern als Polsterung für das Plexiglasrohr. Darüber ist ein beweglicher Rammbär $(g)$ von rund $11 \mathrm{~kg}$ Gewicht aufgesetzt, der erlaubt, das Gerät nach Bedarf einzurammen. Kopf- und Fussteil sind durch stählernde Spannbänder miteinander verbunden, welche das ganze Gerät zusammenhalten. Diese werden am Fussteil mit Hilfe von Schlitzlöchern an runden Zapfen $(h)$ eingehängt; am Kopfteil werden sie mit verstellbaren, im Handel erhältlichen Kistenverschlüssen an Haken (i) eingehängt und zugeklappt. Am unteren Ende sind die Spannbänder mit Distanzstücken versehen, welche den Zentrierring (e) festhalten (in Abb.4 gut sichtbar). Um besonders während des Einrammens eine Durchmischung des überstehenden Wassers im Proberohr möglichst zu vermeiden, sind im Kopfteil zwei leicht bewegliche, halbkreisförmige Klappen $(k)$ eingebaut. Diese stehen horizontal, öffnen sich nach oben und sinken durch ihr Eigengewicht sofort wieder in die Ausgangslage zurück.

Der Verschlussmechanismus wird erst nach dem Bohrvorgang (Stechen oder Rammen) zur Funktion freigegeben. Hierzu dient ein Ausklinkmechanismus, der andern bekannten Einrichtungen nachgebildet ist, der aber, um die grossen Kräfte aufnehmen zu können, erheblich stärker gebaut ist. Er besteht, wie aus Abbildung 2 ersichtlich ist, aus zwei ineinandergeschobenen Stahlrohren von rechteckigem Querschnitt. Das äussere, als Schieber dienende Rohr hält einen Haken aus Stahl fest, der im innern Rohr drehbar gelagert ist und an welchem das Bohrgerät aufgehängt ist. 
Mit einem Fallgewicht wird nun der Haken gelöst, wodurch das Bohrgerät freigegeben wird. Wie bei den übrigen Teilen wurde auch hier darauf geachtet, möglichst einfache, handelsübliche und billige Materialien (Stahl, später verzinkt, ist billiger und stärker als Messing!) und eine einfache, wenig arbeitsaufwendige Konstruktion zu wählen.

\section{Funktion, Betriebsweise}

a) Probenahme. Das Bohrgerät wird mit dem Kopfteil am Ausklinker aufgehängt und mit einer Seilschlaufe zusätzlich gesichert (Abb.3); die Verschlußschaufeln werden ganz geöffnet (Abb.4). Nachdem die Wassertiefe genau ermittelt ist, wird es auf rund $2 \mathrm{~m}$ über Grund abgesenkt und dort kurze Zeit ruhig hängen gelassen, um all-

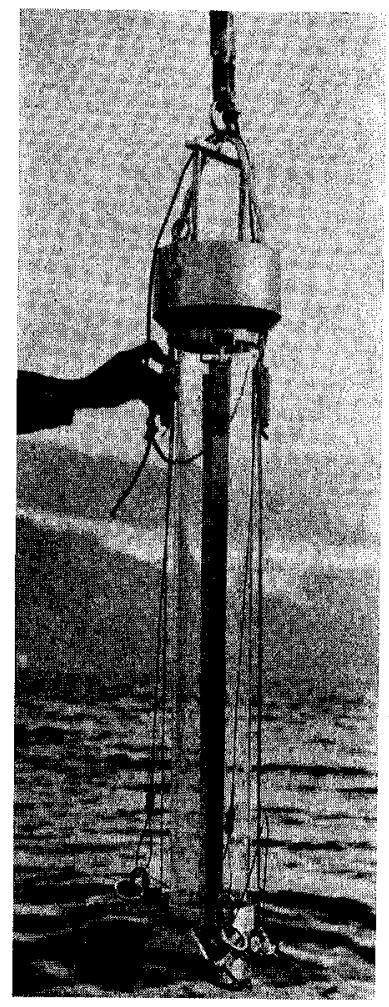

Abb. 3. Gerät, am Auslöser aufgehängt, bereit zur Probenahme.

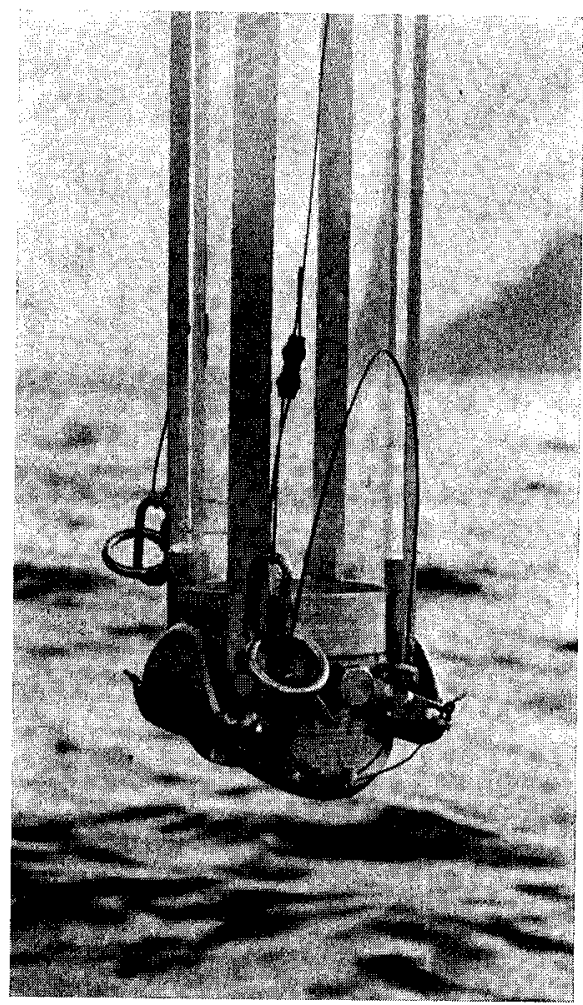

Abb. 4. Fussteil mit geöffneten Verschlußschaufeln. Der Zentrierring wird durch Distanzklötze, auf den Spannbändern aufgeschweisst, gegen oben festgehalten.

fällige Pendelschwingungen verebben zu lassen. Nachher wird es je nach Beschaffenheit des Sediments mehr oder weniger langsam in den Seegrund eingesenkt, frei fallen gelassen oder schliesslich mit dem Rammgewicht noch weiter eingerammt. Unmittelbar nachher wird mit einem Fallgewicht das Gerät ausgeklinkt und hochgezogen. 
Durch das Anziehen des Tragseils werden zuerst die Verschlußschaufeln geschlossen, und erst nachher wird sich das Gerät aus dem Seegrund lösen. Die Tragkraft eines 3-mm-Stahlseils reicht hierzu reichlich aus; hingegen ist für eine genügend kräftige Winde zu sorgen. Das Gerät wird hochgeholt; mit einem Dorn werden die Verschlussschaufeln gesichert, damit sie sich bei den nachfolgenden Manipulationen (bei unbelasteten Zugleinen) nicht öffnen können, und schliesslich wird es ins Boot gehoben. Bei dieser und den nachfolgenden Phasen ist nun grösste Vorsicht geboten: Insbesondere durch rasche Drehbewegungen wird die äusserst empfindliche Sedimentoberfläche leicht aufgerissen und aufgewirbelt. Deshalb wird die frische Probe (Abb.5) nun mit Vorteil sofort an Land gebracht und erst dort präpariert.

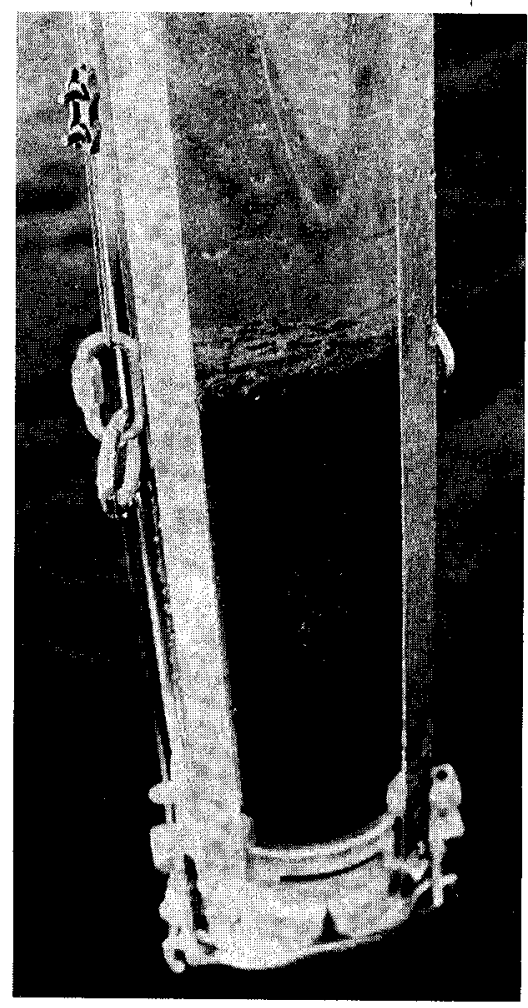

Abb. 5. Bohrkern, unmittelbar nach der Probenahme. Die Schlammoberfläche ist weitgehend intakt, und das überstehende Wasser ist klar.

b) Präparation der Probe. Um das Proberohr bzw. die Probe in ungestörtem Zustand aus dem Gerät herauszunehmen, ist eine einfache Schneideeinrichtung erforderlich. Diese besteht im wesentlichen aus einem verstellbaren, massiv gebauten Tischchen, dessen zweigeteilte, herausnehmbare Platte zwei kreisrunde Ausschnitte für den Fussteil des Gerätes und für den unteren Verschlussdeckel des Proberohrs trägt, ferner einen verstellbaren Support für diesen Deckel (Abb.6). Der im folgenden geschilderte Ablauf der Präparation ist in Abbildung 7 schematisch dargestellt: 


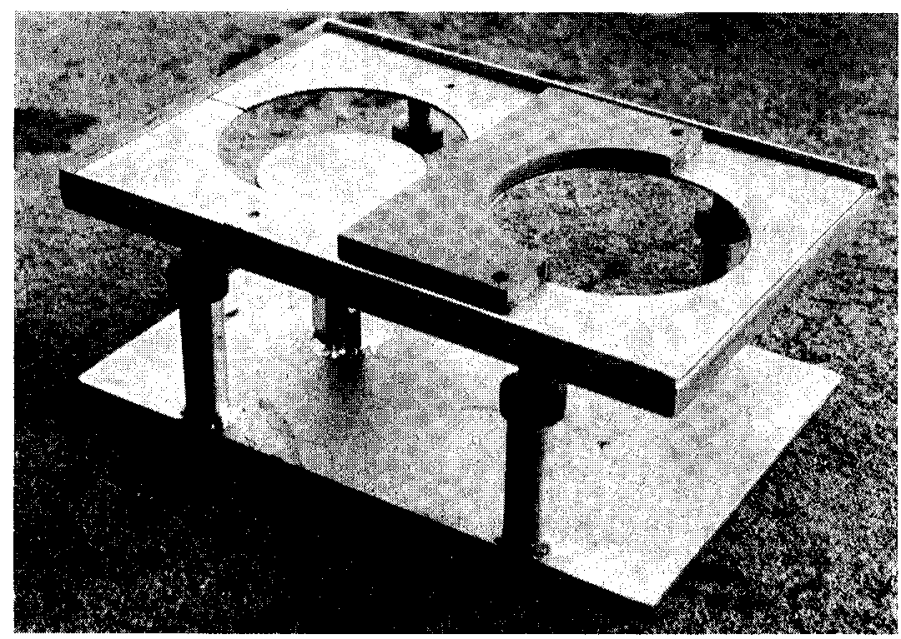

Abb. 6. Schneidetisch mit eingelegter Tischplatte, Haltesegment und Support für den unteren Verschlussdeckel. Tischbeine und Support sind verstellbar.
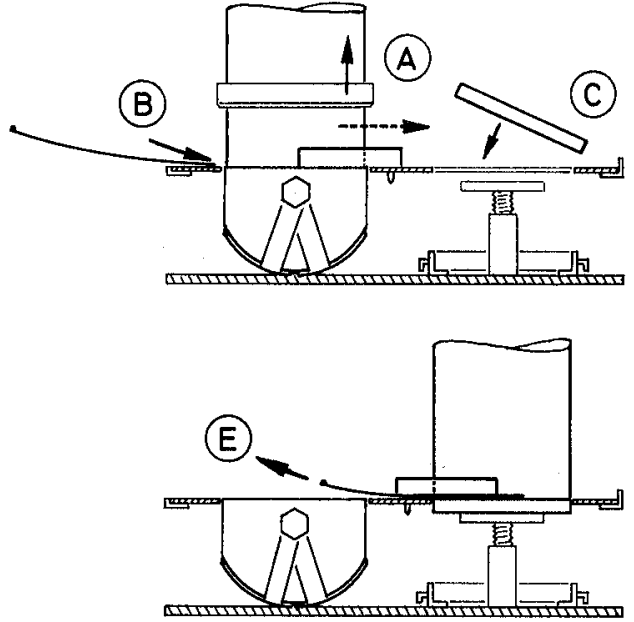
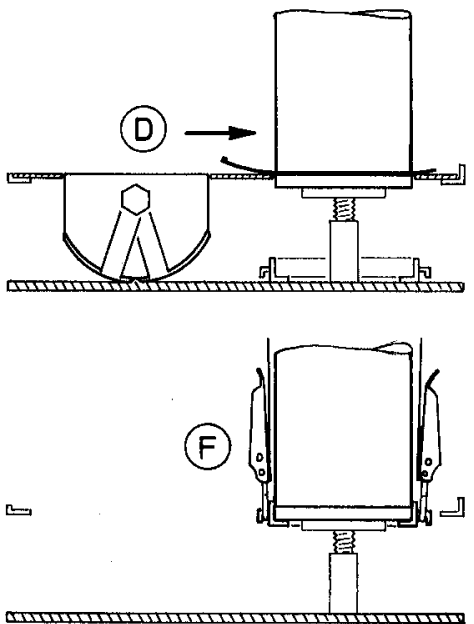

Abb. 7. Schema der Präparation der Probe. Die Beine des Schneidetisches sind in der Zeichnung weggelassen.

Das Gerät wird in den Tischrahmen hineingestellt. Eine Person hält es fest und drückt das Proberohr kräftig nach unten, während die zweite Person die Spannbänder löst und Kopfteil und Zentrierring abhebt (Abb. 7, A). Nun werden die beiden Hälften der Tischplatte in den Rahmen eingelegt. Mit den Stellmuttern, welche an jedem Tischbein vorhanden sind (Abb.6), wird die Tischplatte genau auf die Höhe der Fussoberkante eingestellt. Ein halbkreisförmig ausgeschnittenes, genau auf das Plexirohr passendes Führungssegment, das mit Bolzen in entsprechende Bohrungen der Tischplatte eingesteckt wird (siehe Abb. 6), schützt das Rohr während der folgenden 
Phase davor, weggestossen zu werden. Nun wird ein Stück Blech aus etwa 0,3 mm starker Federbronce sorgfältig zwischen den Fussteil und das Proberohr eingeschoben, bis der ganze Querschnitt durchschnitten ist $(B)$. Das Haltesegment wird hierauf weggenommen. In der zweiten Tischöffnung wird nun der untere Verschlussdeckel auf den Support gelegt und mit Hilfe des schraubbaren Tellers auf die Höhe der Tischplatte eingestellt $(C)$.

Das Schneidblech wird mitsamt dem Proberohr vorsichtig auf die eben vorbereitete Öffnung hinübergeschoben $(D)$. Das Haltesegment wird umgedreht, so dass das Rohr nun in der umgekehrten Bewegungsrichtung fixiert ist. Das Schneidblech wird

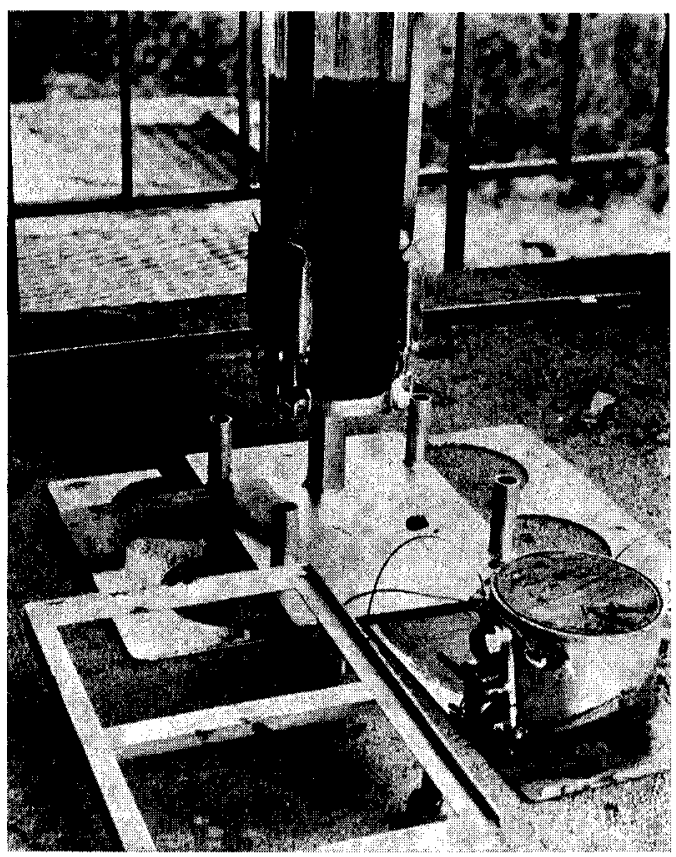

Abb. 8. Fertig präparierte Probe. Vorne der abgehobene Tischrahmen, daneben das Schneidblech und darauf der noch mit Schlamm gefüllte Fuss. Neben der Grundplatte mit der Probe die beiden Hälften der Tischplatte und das Haltesegment. Die Schlammoberfläche ist weitgehend ungestört und das überstehende Wasser klar.

vorsichtig weggezogen, wobei das Haltesegment das Rohr so zentriert, dass es genau auf den unteren Verschlussdeckel zu stehen kommt $(E)$. Das Segment wird nun entfernt, ebenso die beiden Tischplattenhälften. Der obere Deckel wird aufgelegt, der Haltering darübergelegt und die vier Spannbänder, welche zu jedem Rohr gehören, angehängt. Sodann wird der untere Haltering, der schon vor der Präparation eingelegt worden ist, angehoben und mit den Spannbändern, deren Zugkraft noch genau eingestellt werden muss, fixiert $(F)$. Abbildung 8 zeigt eine fertig präparierte Probe (man beachte das klare überstehende Wasser). Damit ist die Probe für die weitere Verwendung im Laboratorium gesichert, und das Gerät kann mit einem neuen Rohr 
für die nächste Probenahme verwendet werden. Für jedes Rohr ist ein entsprechender Zubehörsatz (Verschlussdeckel, Halteringe, vier Spannbänder) notwendig.

Der ganze Apparat ist aus Stahl gebaut und so robust konstruiert, dass er ohne Gefahr für den Verschlussmechanismus mit dem schweren Rammgewicht auch in zähe Sedimente eingerammt werden kann. Sämtliche Teile sind, soweit es sich nicht um rostfreien Stahl handelt (z.B. die Führungsstangen des Rammgewichtes), galvanisch verzinkt.

Das Bohrgerät wurde in der Konstruktionswerkstätte der Versuchsanlage Tüffenwies der EAWAG nach den Plänen des Verfassers hergestellt. Dieser dankt Herrn H.-J. SchÄLCHLI, Werkstattchef, für seine stete Bereitschaft, die Arbeiten kurzfristig ausführen zu lassen, Herrn EUGEN Schwager, Maschinenschlosser, für seine fachkundige und zuverlässige Ausführung des Gerätes und Herrn Dr. RENÉ GächTER für seine Mitwirkung und Kritik bei den verschiedenen Probenahmeversuchen.

\section{Zusammenfassung}

Es wird ein neues Bohrgerät beschrieben, mit welchem ungestörte Sedimentproben von $112 \mathrm{~mm}$ Durchmesser aus beliebigen Wassertiefen gefasst werden können. Der grosse Durchmesser bedingt am unteren Ende des Proberohres einen Verschlussmechanismus, der nach der Probenahme mit einer besonderen Schneideeinrichtung von der Probe abgetrennt wird, ohne diese zu stören.

\section{SUMMARY}

A new method is described for taking sediment sample cores of a diameter of 112 millimeters from deep lakes. The rather large core diameter requires a mechanism which closes the lower end of the core before retraction so that the core can be raised without loss of the sample. The apparatus is set on to a cutting table on which the core, still in the tube, is separated from the closing mechanism without disturbance by means of a blade. The bottom end of the tube is then secured and the corer can be re-used with a new core tube.

\section{LITERATURVERZEICHNIS}

[1] E. Naumann, Einführung in die Bodenkunde der Seen (Stuttgart 1930).

[2] H. ZÜLLIG, Ein neues Lot zur Untersuchung der obersten Schlammschichten, zur Messung des Sedimentabsatzes und zur Evfassung bodennaher Wasserschichten, Schweiz. Z. Hydrol. 15, 275-284 (1953).

[3] S. Ekman, Neue Apparate zur qualitativen und quantitativen Untersuchung der Bodenfauna der Binnenseen, Int. Rev. Hydrobiol. 3, 553-561 (1911).

[4] M. Auerbach, Ein quantitativer Bodengreifer, Beitr. naturk. Forsch. Südw.-Dtl. 12, 17-23 (1953).

Separatdruck aus der Schweiz. Zeitschrift für Hydrologie 31, Fasc. 1 (1969) Herausgegeben mit Unterstützung der Stiftung der Wirtschaft zur Förderung des Gewässerschutzes in der Schweiz 